\title{
Multiple Magnetization Reversal Channels Observed in a 3d-4f Single Molecule Magnet
}

\author{
Asma Amjad ${ }^{1}$, Albert Figuerola ${ }^{2}$, Andrea Caneschi ${ }^{1}$ and Lorenzo Sorace ${ }^{1, *}$ \\ 1 INSTM Research Unit and Dipartimento di Chimica "U. Schiff", Università di Firenze, \\ Sesto Fiorentino (Fl) 50019, Italy; asma.amjad@unifi.it (A.A.); andrea.caneschi@unifi.it (A.C.) \\ 2 Departament de Química Inorgànica i Orgànica, Secció de Química Inorgànica, Universitat de Barcelona, \\ Martí i Franquès 1-11, E-08028 Barcelona, Spain; albert.figuerola@qi.ub.es \\ * Correspondence: lorenzo.sorace@unifi.it; Tel.: +39-055-457-3280
}

Academic Editor: Marius Andruh

Received: 26 April 2016; Accepted: 12 May 2016; Published: 14 June 2016

\begin{abstract}
The present study discusses the magnetic dynamics of a previously reported cyanide bridged 3d-4f dinuclear Dy ${ }^{\mathrm{III}} \mathrm{Co}^{\mathrm{III}}$ complex. Following the axial anisotropy suggested by previous Electron Paramagnetic Resonance spectroscopy (EPR) analysis, the complex turned out to show slow relaxation of the magnetization at cryogenic temperature, and this was studied in different temperature and field regimes. The existence of multichannel relaxation pathways that reverse the magnetization was clearly disclosed: a tentative analysis suggested that these channels can be triggered and controlled as a function of applied static magnetic field and temperature. Persistent evidence of a temperature independent process even at higher fields, attributable to quantum tunneling, is discussed, while the temperature dependent dynamics is apparently governed by an Orbach process. The broad distribution of relaxation rates evidenced by the ac susceptibility measurements suggest a relevant role of the intermolecular interactions in this system.
\end{abstract}

Keywords: lanthanides; slow relaxation of magnetization; single molecules magnets; relaxation pathways

\section{Introduction}

Many interdisciplinary efforts are underway to comprehend the magnetic behavior of lanthanide based molecular magnets (MMs); however, this has proven to be a complex and tangled task owing to the large orbital contribution of lanthanides (large magnetic anisotropy), along with weak exchange interactions and significant crystal field effects normally occurring in such systems [1-5]. The objective of these dedicated attempts is to individually identify and explain the contribution of the aforementioned processes to the static and dynamic magnetic properties of the lanthanide based molecules. Many of these attempts have been performed in mixed d-f metal ion complexes, as a way to bypass the weakness of the $\mathrm{f}-\mathrm{f}$ magnetic exchange interactions. Interestingly, these mixed $\mathrm{d}-\mathrm{f}$ complexes allow the use of the so-called diamagnetic substitution approach, where the transition paramagnetic ion exchange-coupled to the lanthanide ion is substituted with a diamagnetic analogue, at the same time keeping the integrity of the crystal structure. This particular approach provides a qualitative picture of the relative magnitude of the active interactions in the system $[6,7]$ which was particularly precious in the absence of reliable theoretical methods to evaluate them.

On the other hand, more recently, a lot of success has been achieved in understanding the key factors affecting the processes leading to slow relaxation of the magnetization in lanthanide based MMs - much more ubiquitous than initially thought—by use of multiple techniques, including magnetometry, susceptibility, Electron Paramagnetic Resonance spectroscopy (EPR), luminescence and magnetic circular dichroism [8-11], often coupled to detailed $a b$ initio calculations. This provided 
breakthroughs in the understanding of the role of the magnetic anisotropy and the crystal field effects in determining high barriers to the relaxation of magnetization, and provided key inputs for the design and synthesis of compounds showing relatively high magnetization blocking temperatures [12-14]. In this framework, it is interesting to note that most of the systems earlier synthesized for the study of their static magnetic properties have not yet been characterized for the dynamics of their magnetization, despite the few exceptions that have provided clear evidence of their potentialities in this regard $[15,16]$.

The present work is focused on a Dy $\left.\left(\mathrm{H}_{2} \mathrm{O}\right)_{3}(\mathrm{dmf})_{4}(\mu-\mathrm{CN}) \mathrm{Co}-(\mathrm{CN})_{5}\right] \cdot n \mathrm{H}_{2} \mathrm{O}$ where $1 \leqslant n \leqslant 1.5$ and $\mathrm{dmf}=N, N^{\prime}$-dimethylformamide, complex (hereafter DyCo) which had been previously synthesized by one of us [17] to study the competing effect of exchange and anisotropy effects on the static magnetic behavior in a family of isotructural LnM complexes. We underline that, for this derivative, only the crystal field affects the temperature dependence of the susceptibility, given the diamagnetic nature of the low-spin Co ${ }^{\mathrm{III}}$, only slightly affected by temperature independent paramagnetism [17]. The complex has a monoclinic unit cell containing four discrete hetero-dinuclear molecules and crystallizes in the $\mathrm{P} 21 / \mathrm{n}$ (i.e., $\mathrm{n}^{\circ} 14$ ) space group $[7,17]$. A detailed view of the coordination sphere of the Dy ${ }^{\mathrm{II}}$ center is reported as reference in Figure 1: Dy ${ }^{\mathrm{III}}$ coordination number is eight, and analysis of the coordination polyhedron via use of SHAPE [18] provided evidence of a geometry intermediate between square antiprismatic, bicapped trigonal prismatic and trigonal dodecahedron geometry (see Table S1). The analysis of the packing, as well as detailed X-ray investigation on isostructural systems, [19] showed the existence of a 3D network made up of Dy ${ }^{\mathrm{III}}-\mathrm{Co}^{\mathrm{III}}$ dinuclear entities where the five terminal $\mathrm{CN}$ groups of the $\left\{\mathrm{Co}(\mathrm{CN})_{6}\right\}$ fragment are connected through hydrogen bonds to the Ln-coordinated water molecules via crystallization water molecules. The static magnetic properties revealed that this network of hydrogen bonding interaction might result in non-negligible inter-dimer coupling interactions [17].

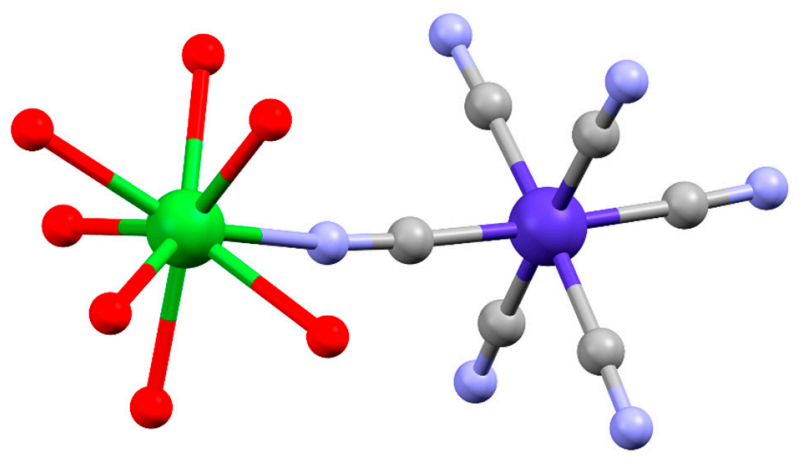

Figure 1. View of the core of DyCo [17]. Color code: blue: Co ${ }^{I I I}$; grey: C; cyan: N; red: O; green: Dy ${ }^{I I I}$.

EPR spectroscopy on a powder sample, performed at $4.2 \mathrm{~K}$ was interpreted under the assumption that the observed properties were only due to the anisotropic ground doublet of Dy ${ }^{\mathrm{III}}$ [20], and suggested that the DyCo boasts an almost axial anisotropy with an effective $g$ tensor $\left(g^{\text {eff }} x, g^{\text {eff }} y\right.$, $\left.g^{e f f} z\right)=(0.1(1), 0.1(1), 18(1))$ [21]. Since axial anisotropy is often accompanied, in Dy-containing systems [22-24], by slow relaxation of the magnetization, this prompted us to study the magnetic dynamics in this complex, which is herein reported as a function of frequency, static magnetic field and temperature. Interestingly, multiple relaxation pathways triggered by wide range of temperature and fields were observed. The different contributions to the slow relaxation of magnetization were then investigated by modeling the experimental data assuming various relaxation mechanisms. Interestingly it was observed that a temperature independent contribution, usually attributed to quantum tunneling of magnetization and thus often ineffective in applied field, survived up to quite a high field. 


\section{Results}

\subsection{Low Field and Temperature Dynamics}

Ac susceptibility as a function of frequency was first performed at the lowest experimentally attainable temperature of $2 \mathrm{~K}$, in the presence of a variable static magnetic field. No out-of-phase component of the susceptibility $\left(\chi^{\prime \prime}\right)$ is observed in zero applied magnetic field (Figure 2a), even if it is quite evident (Figure S1) that a non-zero signal might be present for frequencies above $10 \mathrm{KHz}$ (i.e., outside available experimental range). On application of a static field of 100 Oe a non-zero $\chi^{\prime \prime}$ signal with a clear frequency-dependence is observed, with a maximum around $50 \mathrm{~Hz}$, indicating slow relaxation of magnetization (Figure S1). This behavior is quite common for lanthanide based systems, as a consequence of suppression of quantum tunneling of magnetization (QTM) by an applied magnetic field [25]. Figure 2a shows the behavior of $\chi^{\prime \prime}(v)$ as a function of applied static magnetic field up to $5 \mathrm{KOe}$; it is evident that on increasing field, the magnetization is relaxing slower and at the same time the relaxation times distribution narrows. A more quantitative evaluation of the field dependence of the relaxation rate and its distribution was achieved by fitting the data using the generalized Debye equation [11]:

$$
\chi^{\prime \prime}(w)=\left(\chi_{T}-\chi_{S}\right) \frac{(w \tau)^{1-\alpha} \cos (\pi \alpha / 2)}{1+2(w \tau)^{1-\alpha} \sin (\pi \alpha / 2)+(w \tau)^{2-2 \alpha}}
$$

where $\tau$ is the magnetic relaxation time, $\chi_{\mathrm{T}}$ and $\chi_{\mathrm{S}}$ are the isothermal and adiabatic susceptibility, respectively, while $\alpha$ is a parameter describing the distribution of relaxation times. It is interesting to note that, before the relaxation becomes almost field independent at high fields, a decrease followed by a rise in the relaxation rate is observed from 1.25 to $2.25 \mathrm{KOe}$, Figure $2 \mathrm{~b}$. The field dependence of the $\alpha$ parameter obtained by the Debye fit reveals, as expected, a broad distribution of relaxation times (0.27-0.39) which is essentially field independent above 1 KOe (Figure S2). The observed field dependence of the relaxation rate up to $2 \mathrm{KOe}$ is consistent with the rate being dominated by quantum tunneling processes in zero field, which are then quenched by the application of a field: this in turn increases the efficiency of direct process, resulting in a decrease of $\tau$ at higher fields. Following this interpretation, a phenomenological fit of the data was attempted in this regime using the expression [26]:

$$
\tau^{-1}=\frac{B_{1}}{1+B_{2} * H^{2}}+A_{1} * T H^{4}+C
$$

which provided best fit parameters $\mathrm{B}_{1}=1275 \pm 83 \mathrm{~Hz}, \mathrm{~B}_{2}=(8.8 \pm 0.7) \times 10^{-5} \mathrm{Oe}^{-2}, \mathrm{~A}_{1}=(5 \pm 0.43) \times$ $10^{-13} \mathrm{~Hz}(\mathrm{~K})^{-1} \mathrm{Oe}^{-4}$ and the field independent contribution (either Orbach or Raman), $\mathrm{C}=12.5 \mathrm{~Hz}$.
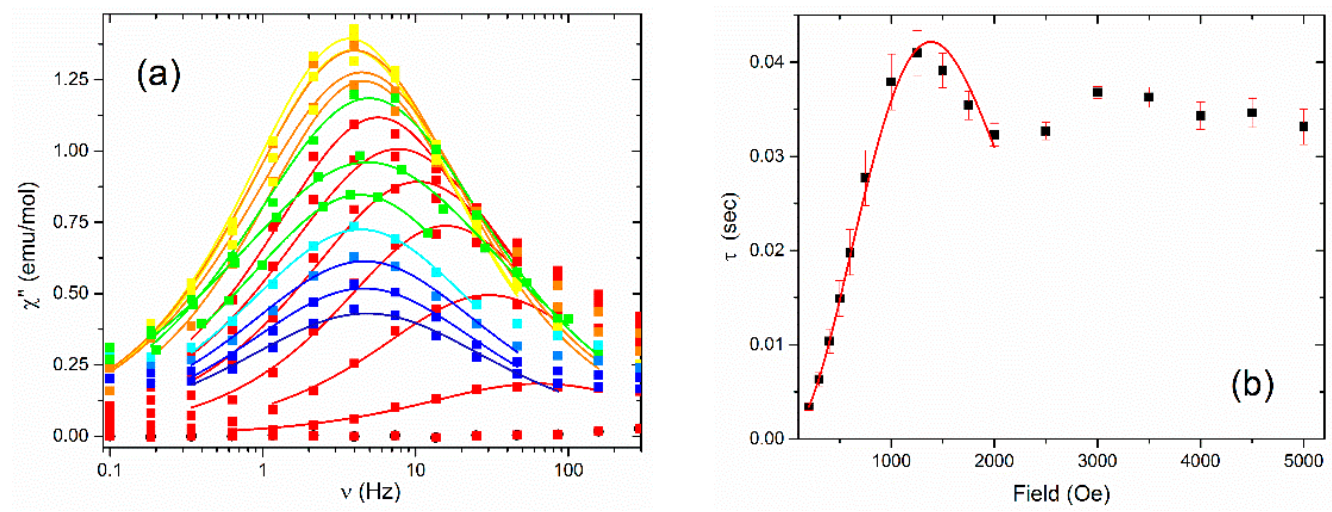

Figure 2. (a) Frequency dependence of the out-of-phase $\chi^{\prime \prime}$ component of the molar AC susceptibility of DyCo measured at $2 \mathrm{~K}$ at variable external static magnetic field (red 0 Oe to blue $5 \mathrm{KOe}$ ). The solid lines are Debye fits at respective fields; (b) Field dependent behavior of the relaxation time observed at $2 \mathrm{~K}$; the solid line is a semi-quantitative fit to the data obtained with parameters reported in the text. 
Existence of multiple processes contributing to relaxation of magnetization in rare-earth based complexes is consistent with the findings in the literature $[23,27,28]$. It is however to be noted that, in the present case, the data clearly indicates that at fields higher than 2 KOe the direct process stops increasing with the field; alternatively, one may assume an exact cancellation between processes the rate of which increases with the field (direct process) and those which should slow down on increasing field (quantum tunneling). Since a small static field was sufficient to trigger slow relaxation of magnetization in this complex, a detailed temperature study was performed in the presence of a static field of $300 \mathrm{Oe}$ to further probe the contribution of different processes to the dynamics. The temperature dependence of the frequency at which the maximum in the imaginary susceptibility component occurs (Figure S3) suggests a single molecular magnetic behavior, as expected for complexes with an axial anisotropic center [29] as is the case here on the basis of previously reported EPR spectroscopy [21].

It is however evident, by a more accurate analysis of the temperature dependence of $\chi(v)$, that at this field two different relaxation phenomena are actually active (Figure S4). At $2 \mathrm{~K}$ indication of one fast $\left(>1.6 \times 10^{-5} \mathrm{~s}\right)$ and one slow $\left(8 \times 10^{-3} \mathrm{~s}\right)$ process can be clearly seen: on increasing temperature the fast process rapidly moves beyond the upper limit of the instrumental frequency range, and simultaneously the slow process become faster and eventually shifts to frequencies out of experimental range at $4 \mathrm{~K}$. The relaxation time of the slower process, derived by the fit to the Debye Equation (1), obeys the Arrhenius law at high temperature. Deviation from linear behavior is evident below $2.2 \mathrm{~K}$, where the relaxation tends toward a temperature independent regime (Figure 3), suggesting the existence of a quantum regime. The respective relaxation time as function of temperature was then modeled using a combination of Arrhenius and quantum tunneling:

$$
\tau^{-1}=D+\tau_{0} \exp ^{\left(-u_{e f f} / k_{B T}\right)}
$$

This provided as best fit parameters, $\tau_{0}=2.38 \times 10^{-9} \mathrm{~s}$ and an effective barrier $U_{\text {eff }}=34 \mathrm{~K}$ $\left(23.87 \pm 0.9 \mathrm{~cm}^{-1}\right)$, with a temperature independent contribution $D$ of $72.36 \pm 10 \mathrm{~Hz}$ which is of the correct order of magnitude compared to what predicted by the fit of field dependent data. Interestingly, a tentative fit performed assuming a combination of Raman $\left(C T^{n}\right)$ and a temperature independent contribution did not provide a meaningful result, the obtained $n$ exponent being far larger than expected (11.8).

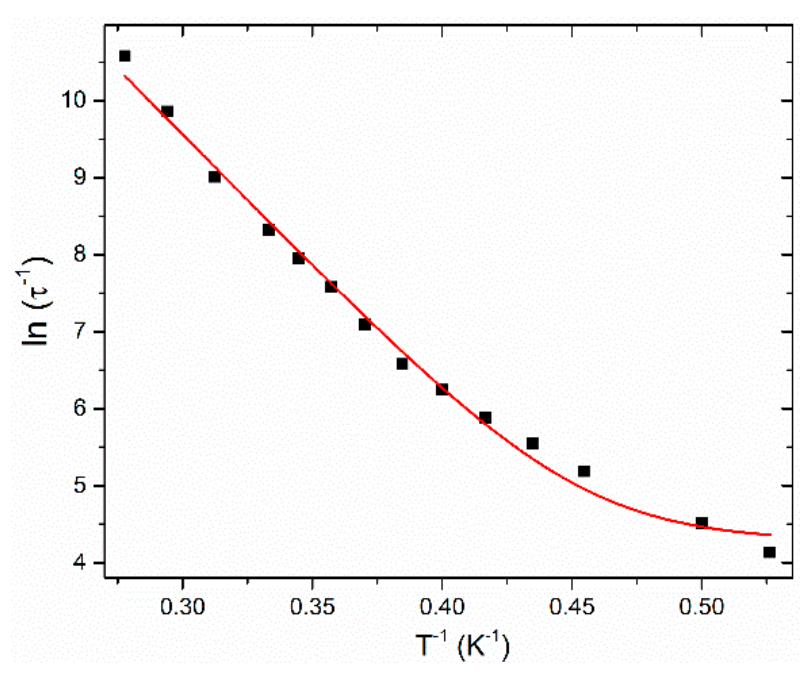

Figure 3. Arrhenius plot of the temperature dependence of the magnetic relaxation time $\tau$ of DyCo, in the presence of a dc magnetic field 300 Oe. The solid line is a fit corresponding to Equation (3) with the best fit parameters reported in the text. 


\subsection{Higher Field Dynamics}

Although relaxation of magnetization was observed to be slowest at about $1.25 \mathrm{KOe}$, a detailed temperature study to observe the evolution of magnetic behavior was performed at higher static applied magnetic field of $2 \mathrm{KOe}$, i.e., in a regime where competing contributions of various relaxation phenomena were observed at low temperature (Figure $2 b$ ). The motivation for this choice lies in exploring the possibility of more easily identifying the multiple relaxation pathways in this compound, already identified in the analysis at 300 Oe. As expected, the data at $2 \mathrm{KOe}$ reveal strong frequency dependence over the whole temperature range (Figure 4a). For temperatures below $2.7 \mathrm{~K}$ a clear maximum (identified as 1 in figure) exists at low frequency; starting from $2.7 \mathrm{~K}$ a broadening of $\chi^{\prime \prime}(v)$ is observed, suggesting the presence of a secondary relaxation (identified as 2 in figure). The second observation is confirmed on increasing temperature by the clearer definition and gradual shift of maximum 2. At intermediate temperatures 3.4-4 K signature of both the peaks is quite clear (Figure 4a). On further increasing temperature up to $8 \mathrm{~K}$ both the maxima completely disappeared and the magnitude of out-of-phase susceptibility decreased to almost zero at $8 \mathrm{~K}$, indicating fast magnetic dynamics in these experimental conditions (Figure S5).
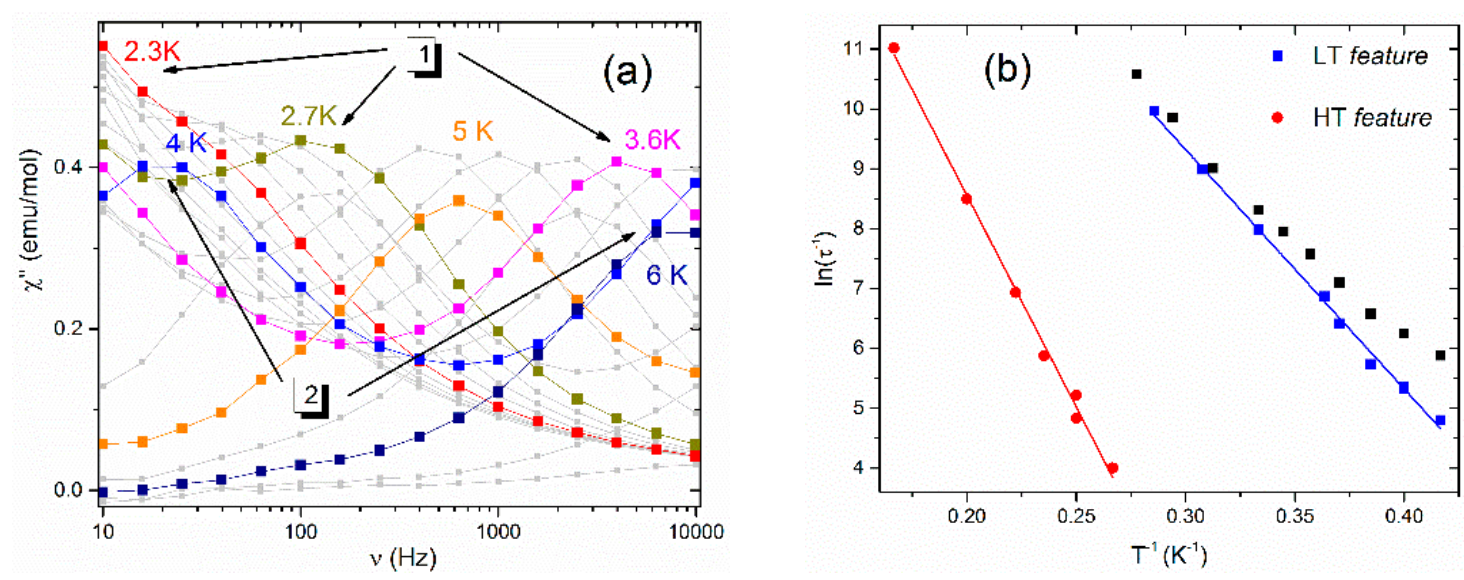

Figure 4. (a) Observation of two identifiable temperature dependent relaxation phenomena in DyCo, in the presence of a static magnetic field of $2 \mathrm{KOe}$; (b) Temperature behavior of the relaxation rates extracted for the two relaxation phenomena from the Debye fit of the ac data at 2 KOe. The solid lines are the best fit to Equation (3) with the parameters explained in the text. Black squares are the relaxation rate observed at 300 Oe, reported here for comparison's sake.

The temperature dependences of the relaxation rates obtained by the fit of $\chi(v)$ are reported as Arrhenius plot in Figure $4 \mathrm{~b}$ : it is evident that for $T>2.5 \mathrm{~K}$ the behavior is similar for the two phenomena, being essentially linear. However, the slope is different, indicating that the anisotropy barrier is different for the two processes. Finally, at low temperature, where only process 1 is visible, a deviation from the linear behavior is observed, indicating a quantum regime. Keeping the above observations in mind, the data was tentatively analyzed using Equation (3), providing the following best fit parameters: $\tau_{0}(1)=(6.2 \pm 1.5) \times 10^{-10} \mathrm{~s}, \mathrm{U}_{\text {eff }}(1)=(39.9 \pm 0.8) \mathrm{K}, \tau_{0}(2)=(1.4 \pm 1) \times 10^{-10} \mathrm{~s}, \mathrm{U}_{\text {eff }}(2)=70.6 \mathrm{~K}$ and a temperature independent term for 1 of $(27 \pm 2) \mathrm{Hz}$. The assumption of two-phonon Raman process contributing to the relaxation in place of Orbach one did not provide reasonable fit for any of the two phenomena.

The slower relaxation phenomenon was further investigated as a function of external applied field at $4 \mathrm{~K}$. In the lower field range an increase in $\chi^{\prime \prime}$ is observed above $10 \mathrm{KHz}$ suggesting the possible presence of a fast relaxation process (as confirmed in Figure S6). An increase in static field magnitude gave rise to a relatively slow process at a frequency of $20 \mathrm{~Hz}$, with further slowing down of magnetization reversal with increasing field as shown in Figure 5a. Upon comparing this 
field-dependent phenomenon to the ones observed at $2 \mathrm{KOe}$, it is quite clear that this is actually the same relaxation phenomenon observed in the 2 KOe data. Interestingly, upon increasing the magnetic field up to $30 \mathrm{KOe}$, the fraction of molecules relaxing via this process decreases till it fades away above $20 \mathrm{KOe}$ (Figure S7). It is significant that while this relaxation channel is quenched, an additional peak appears for fields above $8 \mathrm{KOe}$, indicating the opening of another field triggered relaxation channel. This is a very slow process and it continues to gain intensity and to slow down while digressing outside the experimental range (less than $0.1 \mathrm{~Hz}$ ) at higher fields (Figure S7). Over the whole field range, the Debye modeling of the data yield a range of the $\alpha$ parameter distribution $0.25-0.45$, which indicates a broad distribution of relaxation times.
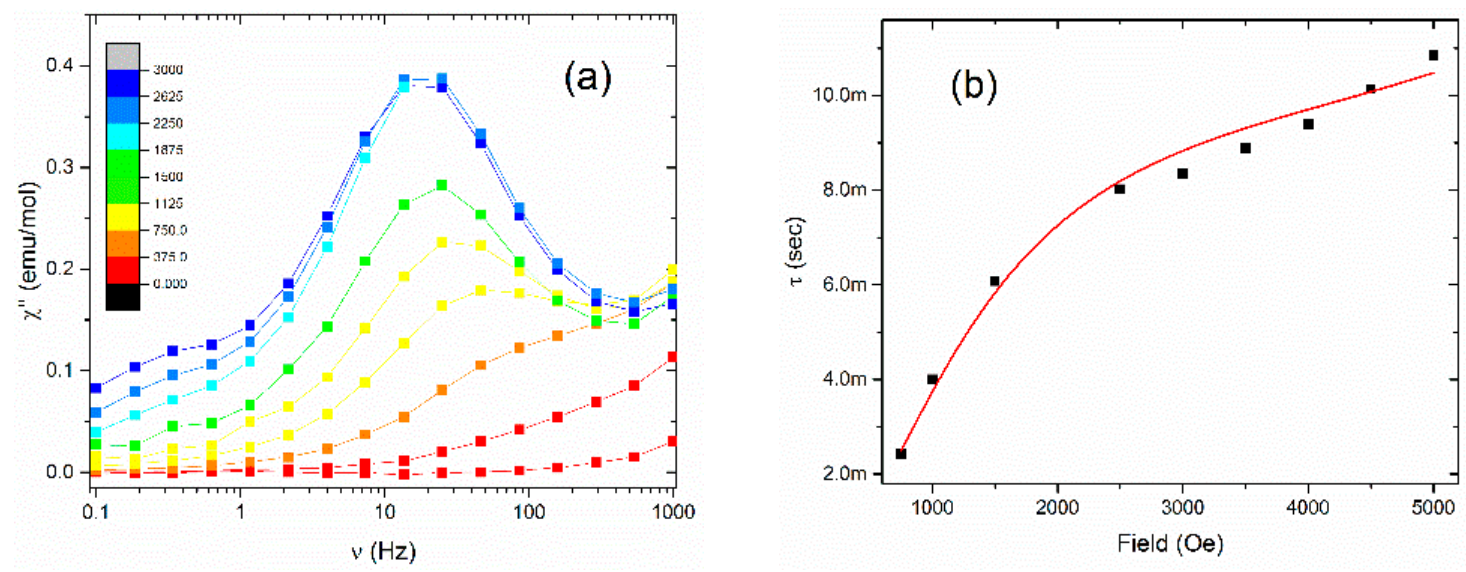

Figure 5. (a) Frequency dependence of the out-of-phase $\chi^{\prime \prime}$ component of the susceptibility measured at $4 \mathrm{~K}$ at variable external static magnetic field. The solid lines are guide for eyes; (b) Field dependent behavior of the relaxation time observed at $4 \mathrm{~K}$, extracted from the Debye fit of the AC data, the solid lines represent the best attainable fit.

The field dependence of the relaxation rate obtained by these data (Figure $5 b$ ) clearly points to a non-linear behavior: for field less than $2.5 \mathrm{KOe}$ a large dependence is observed, followed by a smoother increase above this field. Such behavior suggests that quantum tunneling processes dominates the relaxation, with superimposed contributions from field independent processes like the two-phonon Orbach process (Raman one having been discarded on the basis of the temperature dependence of the relaxation rate). In contrast, the direct term does not appear to play a major role here, since the relaxation time continues to increase up to the highest measured field. The reproduction of relaxation times at $4 \mathrm{~K}$ using the semi-quantitative technique employed for the $2 \mathrm{~K}$ field dependent data verified the aforementioned arguments. The fit procedure performed on the basis of Equation (2), while pointing to a well definite value for the field-independent term $(92.2 \pm 5 \mathrm{~Hz})$, indicated that the absolute value of the parameters phenomenologically describing the QT could not be determined, but only their ratio: best fit was obtained with a ratio $B_{2} / B_{1}=5.71 \times 10^{-8}$, with no appreciable distinction among curves for $\mathrm{B}_{1}>5 \times 10^{5} \mathrm{~Hz}$, which could then be considered as a lower limit. Despite the phenomenological nature of this set of parameters, one can conclude, in agreement with experimental observation, that a significant contribution of quantum tunneling survived even at the highest measured fields of $0.5 \mathrm{~T}$.

\section{Discussion}

Manifestation of multiple relaxation channels is not unusual in lanthanide based complexes [25,30]. However identification and contribution of the individual processes has proven to be quite tricky, due to the complex energetic structure of these ions. Furthermore, analysis of multiple magnetization reversal channels normally requires that each channel is due to a unique or a combination of known relaxation mechanisms like temperature dependent (Orbach, Raman or direct process), temperature 
independent/field-dependent QTM processes and so on. The case of DyCo reported here however presents a different scenario, where the two peaks identified above behave almost identically on increasing temperature, as seen in Figure $4 \mathrm{~b}$. Both the channels apparently undergo reversal of magnetization via the Orbach mechanism, giving an energy barrier of approximately 40 and $70 \mathrm{~K}$, with channel 1 showcasing a small quantum tunneling contribution to relaxation.

Observation of multiple barriers to relaxation in a lanthanide based molecular magnet in a non-varying field is usually attributed to the fact that a certain percentage of the molecules in the cluster behave independently resulting in their own unique dynamics. This could be due to lattice disorder leading to unique species within the complex or the fact that a bunch of molecules resulted in different anisotropy, as first reported for polynuclear SMMs such as $\mathrm{Mn}_{12}$ and its derivatives [31]. In the present case, as mentioned above, the neutral dinuclear units of DyCo are interacting via hydrogen bonds to form a 3D network, involving water molecules from crystallization [7,17]. It is then highly probable for the complex to undergo structure distortion upon partial loss of crystallization water over a length of time, leading to formation of two unique entities within the complex and thus in the observation of two different channels for magnetization reversal [32]. In this sense, since the two processes were observed to contribute almost equally to the total relaxation of magnetization in the complex, as indicated by the relative integrals of the maxima related to the two channels, one can speculate that quantitatively almost equal number of molecules of each species contribute to their relaxation pathways. Furthermore, the relatively broad distribution of relaxation times pictured by the large $\alpha$ values suggests a large disorder involving the two species.

A more astute rationalization of the field and temperature dependence of the two peaks is shown in Figure S8. The data portrayed depicts behavior of the two peaks at 2 and $4 \mathrm{~K}$, both at 0.3 and $2 \mathrm{KOe}$. Comparison of the behavior of the temperature dependence of the relaxation rate confirms that the slow process identified in the low temperature and field dynamics section is peak 1 in Figure $4 \mathrm{a}$, whereas peak 2 represents a phenomenon induced by high field and high temperature (see Figure $4 \mathrm{~b}$ ). Indeed, similar slope (i.e., similar effective barriers) are observed for the measures at the two fields, with only a slight increase in the effective barrier at higher field and a corresponding decrease in the temperature independent contribution [32]. In particular, in the present case, an increase in static field magnitude from 300 Oe to 2 KOe leads to an apparent increase in the effective barrier from 34.4 to $40 \mathrm{~K}$ : however, since we included explicitly temperature independent contributions and direct terms in the fits, the two values should ideally be identical [33]. The observed difference should then not be considered as physically significant but possibly portrays the different weights of other unresolved contributions to the relaxation, varying with the field. Finally, we note that the observation of the slow process on increasing the field at $4 \mathrm{~K}$ may be attributed, in this framework, to a collective process resulting from intermolecular dipolar interactions: a definite proof of this would require, however, the analysis of isostructural YCo sample doped with Dy ${ }^{\mathrm{III}}$, which is beyond the scope of this paper.

\section{Materials and Methods}

\subsection{Synthesis and Structural Characterization}

DyCo was synthesized following the procedure reported in [17]. The purity of the microcrystalline powder used for magnetic dynamic characterization was checked by $\mathrm{X}$-ray powder diffraction carried on a Bruker D8 advance powder diffractometer equipped with a Cu source $\left(\mathrm{K}_{\alpha}, \lambda=1.54 \AA\right.$ ). X-ray powder spectra of the DyCo complex was observed to superimpose with the spectrum obtained from the analysis of the molecular structure obtained by single crystal X-ray diffraction (Figure S9).

\subsection{Magnetic Characterization}

The AC magnetic susceptibility measurements were carried out on a Quantum Design MPMS SQUID magnetometer and Quantum Design PPMS in AC mode at both zero and applied external DC field in the presence of 5 Oe oscillating magnetic field. The later was used for high frequencies of 
$10 \mathrm{~Hz}$ to $10 \mathrm{KHz}$ whereas the former was employed for more sensitive measurements $0.1 \mathrm{~Hz}$ to $1 \mathrm{KHz}$. All the measurements were executed on polycrystalline sample pressed in a pellet (diameter $5 \mathrm{~mm}$ ) to avoid individual field alignment of the crystallites. Raw data were reduced to paramagnetic molar susceptibilities using molecular formula obtained by X-ray data. This also allowed for evaluation of the intrinsic diamagnetism of the sample by use of corresponding Pascal's constants. As a further purity check, dc magnetic data were measured on the same pellet (Figure S10), and turned out to be in agreement with previously reported characterization [17].

\section{Conclusions}

We reported here a new example of slowly relaxing Dy ${ }^{\mathrm{III}}$ based complex, which has been selected on the basis of previously reported static magnetic characterization. The analysis of its dynamic magnetic properties clearly demonstrates the need for detailed field and temperature dependent studies to disentangle the different contributions to the relaxation. In particular, three different processes have been identified, of which one is attributed to a collective process resulting from the network of intermolecular interactions characterizing these systems, and two single molecular ones, which may arise from the partial loss of crystallization solvent. Despite this problematic issue, we feel that this family of cyano-bridged 3d-4f complexes should be further investigated, in view of the interesting photo-switchable properties of some of its class, up to relatively high temperature [34,35] which may open perspective applications in the fields of photo-controlled switches and information storage.

Supplementary Materials: The following are available online at www.mdpi.com/2312-7481/2/2/27/s1; Results of agreement factors for different eight-coordination polyhedra as obtained by SHAPE (Table S1). Additional AC characterization; PXRD spectrum and dc magnetic characterization (Figures S1-S10).

Acknowledgments: We acknowledge the financial support of Italian MIUR through the project Futuro in Ricerca 2012 (RBFR12RPD1).

Author Contributions: A.F. prepared the complex under discussion; A.A. performed the experiment and wrote the paper, L.S. and A.A. analyzed and discussed the experimental data. All the authors reviewed the paper.

Conflicts of Interest: The authors declare no conflict of interest.

\section{Abbreviations}

The following abbreviations are used in this manuscript:

SMM Single Molecule Magnet

QTM Quantum Tunneling of Magnetization

$\mathrm{AC}$ and DC Alternating and Direct current

EPR Electron Paramagnetic Resonance

\section{References}

1. Rinehart, J.D.; Long, J.R. Exploiting single-ion anisotropy in the design of f-element single-molecule magnets. Chem. Sci. 2011, 2, 2078-2085. [CrossRef]

2. Zhang, P.; Guo, Y.; Tang, J. Recent advances in dysprosium-based single molecule magnets: Structural overview and synthetic strategies. Coord. Chem. Rev. 2013, 257, 1728-1763. [CrossRef]

3. Benelli, C.; Gatteschi, D. Introduction to Molecular Magnetism, from Transition Metals to Lanthanides; Wiley-VCH Verlag GmbH \& Co.: Weinheim, Germany, 2015; pp. 239-250.

4. Lucaccini, E.; Briganti, M.; Perfetti, M.; Vendier, L.; Costes, J.-P.; Totti, F.; Sessoli, R.; Sorace, L. Relaxation Dynamics and Magnetic Anisotropy in a Low-Symmetry Dy ${ }^{\mathrm{III}}$ Complex. Chem. Eur. J. 2016, 22, 5552-5562. [CrossRef] [PubMed]

5. Pointillart, F.; Jung, J.; Berraud-Pache, R.; Le Guennic, B.; Dorcet, V.; Golhen, S.; Cador, O.; Maury, O.; Guyot, Y.; Decurtins, S.; et al. Luminescence and Single-Molecule Magnet Behavior in Lanthanide Complexes Involving a Tetrathiafulvalene-Fused Dipyridophenazine Ligand. Inorg. Chem. 2015, 54, 5384-5397. [CrossRef] [PubMed] 
6. Lukens, W.W.; Walter, M.D. Quantifying Exchange Coupling in f-Ion Pairs Using the Diamagnetic Substitution Method. Inorg. Chem. 2010, 49, 4458-4465. [CrossRef] [PubMed]

7. Sorace, L.; Sangregorio, C.; Figuerola, A.; Benelli, C.; Gatteschi, D. Magnetic Interactions and Magnetic Anisotropy in Exchange Coupled $4 \mathrm{f}-3 \mathrm{~d}$ Systems: A Case Study of a Heterodinuclear $\mathrm{Ce}^{3+}-\mathrm{Fe}^{3+}$ Cyanide-Bridged Complex. Chem. Eur. J. 2009, 15, 1377-1388. [CrossRef] [PubMed]

8. Pedersen, K.S.; Woodruff, D.N.; Bendix, J.; Clérac, R. Lanthanides and Actinides in Molecular Magnetism; Layfield, R., Murugesu, M., Eds.; Wiley-VCH: Weinheim, Germany, 2015; pp. 125-152.

9. Gysler, M.; El Hallak, F.; Ungur, L.; Marx, R.; Hakl, M.; Neugebauer, P.; Rechkemmer, Y.; Lan, Y.; Sheikin, I.; Orlita, M.; et al. Multitechnique investigation of $\mathrm{Dy}_{3}$-Implications for coupled lanthanide clusters. Chem. Sci. 2016. [CrossRef]

10. Moreno Pineda, E.; Chilton, N.F.; Marx, R.; Dörfel, M.; Sells, D.O.; Neugebauer, P.; Jiang, S.-D.; Collison, D.; van Slageren, J.; McInnes, E.J.L.; et al. Direct measurement of dysprosium(III)-dysprosium(III) interactions in a single-molecule magnet. Nat. Commun. 2014, 5, 5243. [CrossRef] [PubMed]

11. Gatteschi, D.; Sessoli, R.; Villain, J. Molecular Nanomagnets; Oxford University Press: Oxford, UK, 2006.

12. Liu, J.; Chen, Y.; Jia, J.; Liu, J.; Vieru, V.; Ungur, L.; Chibotaru, L.F.; Lan, Y.; Wernsdorfer, W.; Gao, S.; et al. A Stable Pentagonal-Bipyramidal Dy(III) Single-Ion Magnet with a Record Magnetization Reversal Barrier over 1000 K. J. Am. Chem. Soc. 2016, 138, 5441-5450. [CrossRef] [PubMed]

13. Feltham, H.L.C.; Brooker, S. Review of purely $4 \mathrm{f}$ and mixed-metal nd-4f single-molecule magnets containing only one lanthanide ion. Coord. Chem. Rev. 2014, 276, 1-33. [CrossRef]

14. Rinehart, J.D.; Fang, M.; Evans, W.J.; Long, J.R. Strong exchange and magnetic blocking in $\mathrm{N}_{2}{ }^{3-}$-radical-bridged lanthanide complexes. Nat. Chem. 2011, 3, 538-542. [CrossRef] [PubMed]

15. Kritikos, M.; Moustiakimov, M.; Wijk, M.; Westin, G. Synthesis, structure and characterization of $\mathrm{Ln}_{5} \mathrm{O}(\mathrm{OPri})_{13}$ with $\mathrm{Ln}=\mathrm{Nd}$, Gd or Er. J. Chem. Soc. Dalton Trans. 2001, 1931-1938. [CrossRef]

16. Blagg, R.J.; Muryn, C.A.; McInnes, E.J.L.; Tuna, F.; Winpenny, R.E.P. Single Pyramid Magnets: Dy5 Pyramids with Slow Magnetic Relaxation to $40 \mathrm{~K}$. Angew. Chem. Int. Ed. 2011, 50, 6530-6533. [CrossRef] [PubMed]

17. Figuerola, A.; Diaz, C.; Ribas, J.; Tangoulis, V.; Granell, J.; Lloret, F.; Mahía, J.; Maestro, M. Synthesis and Characterization of Heterodinuclear $\mathrm{Ln}^{3+}-\mathrm{Fe}^{3+}$ and $\mathrm{Ln}^{3+}-\mathrm{Co}^{3+}$ Complexes, Bridged by Cyanide Ligand $\left(\mathrm{Ln}^{3+}=\right.$ Lanthanide Ions). Nature of the Magnetic Interaction in the $\mathrm{Ln}^{3+}-\mathrm{Fe}^{3+}$ Complexes. Inorg. Chem. 2003, 42, 641-649. [CrossRef] [PubMed]

18. Casanova, D.; Llunel, M.; Alemany, P.; Alvarez, S. The Rich Stereochemistry of Eight-Vertex Polyhedra: A Continuous Shape Measures Study. Chem. Eur. J. 2005, 11, 1479-1494. [CrossRef] [PubMed]

19. Svendsen, H.; Overgaard, J.; Chevallier, M.; Collet, E.; Iversen, B.B. Photomagnetic Switching of the Complex $\left[\mathrm{Nd}(\mathrm{dmf})_{4}\left(\mathrm{H}_{2} \mathrm{O}\right)_{3}(\mu-\mathrm{CN}) \mathrm{Fe}(\mathrm{CN})_{5}\right] \cdot \mathrm{H}_{2} \mathrm{O}$ Analyzed by Single-Crystal X-Ray Diffraction. Angew. Chem. Int. Ed. 2009, 48, 2780-2783. [CrossRef] [PubMed]

20. Abragam, A.; Bleaney, B. Electron Paramagnetic Resonance of Transition Ions; Dover Publications: New York, NY, USA, 1986; pp. 276-345.

21. Tangoulis, V.; Figuerola, A. Anisotropic exchange interactions in $\mathrm{Ln}^{3+}-\mathrm{Fe}^{3+} \mathrm{LS}$ dinuclear systems $\left(\mathrm{Ln}^{3+}=\mathrm{Dy}_{\text {, }}\right.$ $\mathrm{Tm}, \mathrm{Yb}$ ): Magnetometry and Dual Mode X-band Electron Paramagnetic Resonance spectroscopic study. Chem. Phys. 2007, 340, 293-301. [CrossRef]

22. Sorace, L.; Benelli, C.; Gatteschi, D. Lanthanides in molecular magnetism: Old tools in a new field. Chem. Soc. Rev. 2011, 40, 3092-3104. [CrossRef] [PubMed]

23. Liddle, S.T.; van Slageren, J. Improving f-element single molecule magnets. Chem. Soc. Rev. 2015, 44, 6655-6669. [CrossRef] [PubMed]

24. Jeletic, M.; Lin, P.; Le Roy, J.J.; Korobkov, I.; Gorelsky, S.I.; Murugesu, M. An Organometallic Sandwich Lanthanide Single-Ion Magnet with an Unusual Multiple Relaxation Mechanism. J. Am. Chem. Soc. 2011, 133, 19286-19289. [CrossRef] [PubMed]

25. Habib, F.; Long, J.; Lin, P.; Korobkov, I.; Ungur, L.; Wernsdorfer, W.; Chibotaru, L.F.; Murugesu, M. Supramolecular architectures for controlling slow magnetic relaxation in field-induced single-molecule magnets. Chem. Sci. 2012, 3, 2158-2164. [CrossRef]

26. Lucaccini, E.; Sorace, L.; Perfetti, M.; Costes, J.; Sessoli, R. Beyond the anisotropy barrier: Slow relaxation of the magnetization in both easy-axis and easy-plane $\mathrm{Ln}$ (trensal) complexes. Chem. Commun. 2014, 50, 1648-1651. [CrossRef] [PubMed] 
27. Fukuda, T.; Shigeyoshi, N.; Yamamura, T.; Ishikawa, N. Magnetic Relaxations Arising from Spin-Phonon Interactions in the Nonthermally Activated Temperature Range for a Double-Decker Terbium Phthalocyanine Single Molecule Magnet. Inorg. Chem. 2014, 53, 9080-9086. [CrossRef] [PubMed]

28. Car, P.E.; Perfetti, M.; Mannini, M.; Favre, A.; Caneschi, A.; Sessoli, R. Giant field dependence of the low temperature relaxation of the magnetization in a dysprosium(III)-DOTA complex. Chem. Commun. 2011, 47, 3751-3753. [CrossRef] [PubMed]

29. Gregson, M.; Chilton, N.F.; Ariciu, A.-M.; Tuna, F.; Crowe, I.F.; Lewis, W.; Blake, A.J.; Collison, D.; McInnes, E.L.J.; Winpenny, R.E.P.; et al. A Monometallic Lanthanide Bis(methanediide) Single Molecule Magnet with a Large Energy Barrier and Complex Spin Relaxation Behaviour. Chem. Sci. 2016, 7, 155-165. [CrossRef]

30. Blagg, R.J.; Ungur, L.; Tuna, F.; Speak, J.; Comar, P.; Collison, D.; Wernsdorfer, W.; McInnes, E.J.L.; Chibotaru, L.F.; Winpenny, R.E.P. Magnetic relaxation pathways in lanthanide single-molecule magnets. Nat. Chem. 2013, 5, 673-678. [CrossRef] [PubMed]

31. Evangelisti, M.; Bartolomé, J. Secondary magnetic relaxations in $\mathrm{Mn}_{12}$ complexes. J. Magn. Magn. Mater. 2000, 221, 99-102. [CrossRef]

32. Aubin, S.M.J.; Sun, Z.; Guzei, I.A.; Rheingold, A.L.; Christou, G.; Hendrickson, D.N. Single-molecule magnets: isomeric $\left[\mathrm{Mn}_{12} \mathrm{O}_{12}\left(\mathrm{O}_{2} \mathrm{CC}_{6} \mathrm{H}_{4} \mathrm{Me}-4\right)_{16}\left(\mathrm{H}_{2} \mathrm{O}\right)_{4}\right]$ complexes exhibiting different rates of resonant magnetization tunnelling. Chem. Commun. 1997, 22, 2239-2240. [CrossRef]

33. Amjad, A.; Madalan, A.M.; Andruh, M.; Caneschi, A.; Sorace, L. Slow relaxation of magnetization in an Isostructural series of Zinc-lanthanide complexes: An integrated EPR and AC susceptibility study. Chem. Eur. J. 2016. [CrossRef]

34. Li, G.; Akitsu, T.; Sato, O.; Einaga, Y. Photoinduced Magnetization of the Cyano-Bridged 3d-4f Heterobimetallic Assembly $\mathrm{Nd}(\mathrm{DMF})_{4}\left(\mathrm{H}_{2} \mathrm{O}\right)_{3}(\mu-\mathrm{CN}) \mathrm{Fe}(\mathrm{CN})_{5} \cdot \mathrm{H}_{2} \mathrm{O}(\mathrm{DMF}=N, N$-Dimethylformamide $)$. J. Am. Chem. Soc. 2003, 125, 12396-12397. [CrossRef] [PubMed]

35. Svendsen, H.; Jørgensen, M.R.; Overgaard, J.; Chen, Y.; Chastanet, G.; Létard, J.; Kato, K.; Takata, M.; Iversen, B.B. Analysis of the Photomagnetic Properties of Cyano-Bridged Heterobimetallic Complexes by X-Ray Diffraction. Inorg. Chem. 2011, 50, 10974-10984. [CrossRef] [PubMed]

(C) 2016 by the authors; licensee MDPI, Basel, Switzerland. This article is an open access article distributed under the terms and conditions of the Creative Commons Attribution (CC-BY) license (http://creativecommons.org/licenses/by/4.0/). 